\title{
Numerical Shallow Water Simulation Using Godunov-Type Scheme on Quadtree Grids
}

\author{
Masayuki Fujihara'
}

\begin{abstract}
This paper deals with a second order accurate Godunov-type numerical model of the two-dimensional shallow water equations discretized using finite volumes. Roe's flux function is used for the convection terms, and a non-linear limiter is applied to prevent non-physical oscillations. The mathematical formulation of shallow water equation, used in this paper, is suitable for cases where the bathymetry is non-uniform. The model is based on hierarchical quadtree grids automatically generated to fit the boundary configuration. A hydraulic jump and a tidal wave propagation into a channel with uneven bottom are simulated as validation tests and then the flows over a cascadetype head work and through a vertical slot-type fishway are computed as applications.
\end{abstract}

\section{Introduction}

In recent years, the number of papers devoted to the numerical simulation of shallow water equations with Riemann solvers has increased (Alcrude and GarciaNavarro, 1993; Zhao et al., 1994; Zhao et al., 1996; Anastasiou and Chan, 1997; Hu et al., 1998; Mingham and Causon, 1998). Roe's scheme (Roe, 1981) used in this paper is one of the approximate Riemann solvers (Toro, 1999), which are applicable to both the subcritical and supercritical flows and also can clearly capture a discontinuous front, such as a hydraulic jump or a dambreak flow.

On the other hand, considering the complicated natural shallow water regions, it is also important for numerical models to generate computational grids fitting into the flow boundaries correctly. Structured grids based on boundary-fitted curvilinear systems have been widely used for the models of natural rivers and coastal areas to reproduce land boundaries correctly (Lin and Falconer, 1995; Borthwick and Akponasa, 1997). For flow-fields with multiple bodies, however, it is particularly difficult to generate structured grids. In the past several years, unstructured grid methods, which are commonly used in finite element methods, have become more and more prevalent even in finite difference modelings. Generally speaking, grid generators ideally should be automatic and robust, and fit the flow boundaries correctly. Hierarchical grid generation appears to be an attractive option in that it meets most of the foregoing requirements. Two-dimensional quadtree grids used in this paper (Greaves and Borthwick, 1998) may be produced extremely quickly by simple spatial decomposition into squares.

The work presented consists of a grid generation based on quadtree grids, and a flow solver based on the MUSCL concept with slope limiter and Roe's approximate Riemann solver (Toro, 1999). The shallow water equations are discretized using finite volumes collocated with the grid cells, and integrated in time using a 4th order Runge-Kutta scheme. Two validation tests designed to verify the accuracy of the model are implemented. The tests include simulations of a hydraulic jump on a horizontal bottom and a tidal wave propagation into a channel with non-uniform bathymetry. In

\footnotetext{
'Associate Professor, Faculty of Agriculture, Ehime University, 3-5-7 Tarumi, Matsuyama, 790-8566 Japan
}

addition, two application tests are presented; the flow over a cascade type head work and the flow through a vertical slot type fishway.

\section{Quadtree grid generation}

Quadtree grids offer a high resolution locally refined mesh consisting of quadrilateral cells. Quadtree grids are generated by recursive subdivision of cells to a level determined by a prescribed subdivision criterion. This technique was originally used for spatial decomposition of digitised images. The method has since been used in the flow simulation as an efficient use of computer storage and for data handling.

The steps to generate a quadtree grid for a maximum division level equal to $M_{\max }$ may be summarised as follows (Greaves and Borthwick, 1998).

1. Define the set of seeding points $P_{n}$ which are usually on the perimeter to represent the boundary geometry.

2. Define a unit square (root panel) which surrounds the domain of interest.

3. Divide the root panel into four quadrant panels.

4. Consider each panel in turn; if the panel contains a seeding point, continue with step 5; otherwise check the next panel.

5. Check whether the maximum division level $M_{\max }$ has been reached. If so, the division of the panel in question is completed. Therefore, go to step 4 and check the next panel. When all panels considered have reached the maximum division $M_{m u x}$, the mesh generation is completed. Otherwise continue.

6. Divide the panel into four congruent panels, return to step 4 and check the next panel.

7. Finally, impose regularisation process, which is achieved by ensuring that the panel edge length ratio between two adjacent panels does not exceed two.

Each cell is given a unique identification number consisting of a concatenated series of $1 \mathrm{~s}$ and $2 \mathrm{~s}$ according to its position in the unit square. The identification number is built-up from the root (i.e., unit square) level during the subdivision process; at any arbitrary level the numbers $11,12,22$, and 21 refer to north-west, northeast, south-east and south-west respectively.

Cell neighbour finding is undertaken, making use of the quadtree structure. It is straightforward to determine the identification numbers of adjacent cells through simple manipulations of the identification number. 


\section{Governing equations}

The shallow water equations, which describe flow in shallow water bodies where the vertical acceleration within the fluid is negligible, can be written as

$$
\begin{gathered}
\frac{\partial \zeta}{\partial t}+\frac{\partial(u h)}{\partial x}+\frac{\partial(v h)}{\partial y}=0 \\
\frac{\partial(u h)}{\partial t}+\frac{\partial\left(u^{2} h\right)}{\partial x}+\frac{\partial(u v h)}{\partial y}-v\left(\frac{\partial\left(h u_{x}\right)}{\partial x}+\frac{\partial\left(h u_{y}\right)}{\partial y}\right) \\
=\frac{\tau_{w x}-\tau_{b x}}{\rho}-g h \frac{\partial \zeta}{\partial x}+h f v \\
\frac{\partial(v h)}{\partial t}+\frac{\partial(u v h)}{\partial x}+\frac{\partial\left(v^{2} h\right)}{\partial y}-v\left(\frac{\partial\left(h v_{x}\right)}{\partial x}+\frac{\partial\left(h v_{y}\right)}{\partial y}\right) \\
=\frac{\tau_{w y}-\tau_{b y}}{\rho}-g h \frac{\partial \zeta}{\partial y}-h f u
\end{gathered}
$$

where $\zeta$ is the free surface elevation above the still water level $h_{s}, h\left(=\zeta+h_{s}\right)$ the total water depth, $u$ and $v$ the depth-averaged velocities in the $x$ - and $y$-directions respectively, $u_{x}, u_{y}$ and $v_{x}, v_{y}$ the derivatives of the depth-averaged velocity components in the $x$ - and $y$ directions respectively, $g$ the acceleration due to gravity, $\rho$ the water density, $\tau_{w x}$ and $\tau_{w y}$ the surface stresses, $\tau_{b x}$ and $\tau_{b y}$ the bed friction stresses, $f$ the Coriolis parameter and $v$ the kinematic eddy viscosity coefficient.

The $g h \partial \zeta / \partial x$ term is usually split to give the hyperbolic system used by many researchers (e.g., Toro, 1999). However, when applied to non-uniformly varying bathymetries, this approach leads to problems nonconserving mass and momentum for individual cells when using Roe's approximate Riemann solver (Nujic', 1995). Thus, in this paper the gh $\partial \zeta / \partial x$ term and similarly the $g h \partial \zeta / \partial y$ term are split differently so that the shallow water equations are written as (Rogers et al., in press)

$$
\begin{gathered}
\frac{\partial \zeta}{\partial t}+\frac{\partial(u h)}{\partial x}+\frac{\partial(v h)}{\partial y}=0 \\
\frac{\partial(u h)}{\partial t}+\frac{\partial\left(u^{2} h+\frac{1}{2} g\left(\zeta^{2}+2 \zeta h_{s}\right)\right)}{\partial x}+\frac{\partial(u v h)}{\partial y} \\
-v\left(\frac{\partial\left(h u_{x}\right)}{\partial x}+\frac{\partial\left(h u_{y}\right)}{\partial y}\right)=\frac{\tau_{w x}-\tau_{b x}}{\rho}-g \zeta S_{o x}+h f v \\
\frac{\partial(v h)}{\partial t}+\frac{\partial(u v h)}{\partial x}+\frac{\partial\left(v^{2} h+\frac{1}{2} g\left(\zeta^{2}+2 \zeta h_{s}\right)\right)}{\partial y} \\
-v\left(\frac{\partial\left(h v_{x}\right)}{\partial x}+\frac{\partial\left(h v_{y}\right)}{\partial y}\right)=\frac{\tau_{u y}-\tau_{b y}}{\rho}-g \zeta S_{o y}-h f u
\end{gathered}
$$

where $S_{o x}$ and $S_{o y}$ are the bed slopes in the $x$ - and $y$ directions, respectively. This formulation allows Roe's approximate Riemann solver to be used to evaluate the inviscid flux between adjacent cells for all bathymetric conditions.

\section{Flow solver}

The two-dimensional shallow water equations are discretized spatially using finite volumes on the quadtree grid, with Roe's flux function used to approximate the non-linear convection terms. Time integration is implemented by means of the 4th order Runge-Kutta integration scheme. Unwanted oscillations are avoided using a slope limiter.

The shallow water equations can be written in integral form as

$$
\frac{\partial}{\partial t} \int_{\Omega} \mathbf{q} \mathrm{d} \Omega+\int_{\Omega}\left(\frac{\partial \mathbf{f}}{\partial x}+\frac{\partial \mathbf{g}}{\partial y}\right) \mathrm{d} \Omega=\int_{\Omega} \mathbf{h d} \Omega
$$

where $\Omega$ is the domain of interest, $\mathrm{q}$ the vector of conserved variables, $f$ and $g$ the flux vectors, and $h$ the vector of forcing functions. The vectors $q, f, g$, and $h$ are given by

$$
\begin{gathered}
\mathbf{q}=\left[\begin{array}{c}
\zeta \\
u h \\
v h
\end{array}\right], \quad \mathbf{f}=\left[\begin{array}{c}
u h \\
u^{2} h+g\left(\zeta^{2}+2 \zeta h_{s}\right) / 2-v h \partial u / \partial x \\
u v h-v h \partial v / \partial x
\end{array}\right] \\
\mathbf{g}=\left[\begin{array}{c}
v h \\
v^{2} h+g\left(\zeta^{2}+2 \zeta h_{s}\right) / 2-v h \partial v / \partial y
\end{array}\right]
\end{gathered}
$$

and

$$
\mathbf{h}=\left[\begin{array}{c}
0 \\
\left(\tau_{w x}-\tau_{b x}\right) / \rho-g \zeta S_{o x}+h f v \\
\left(\tau_{w y}-\tau_{b y}\right) / \rho-g \zeta S_{o y}-h f u
\end{array}\right]
$$

(3) can be written as

$$
\frac{\partial}{\partial t} \int_{\Omega} \mathbf{q} \mathrm{d} \Omega+\oint_{S} \hat{\mathbf{f}} \mathrm{d} S=\int_{\Omega} \mathbf{h d} \Omega
$$

where $S$ is the boundary of $\Omega$, and $\hat{\mathbf{f}}$ the vector of flux functions through $S$.

The term $\hat{\mathbf{f}}$ may also be written in terms of inviscid and viscous fluxes as

$$
\hat{\mathbf{f}}=\mathbf{f}^{1}-v \mathbf{f}^{\mathrm{v}}
$$

with

$$
\mathbf{f}^{\prime}=\left[\begin{array}{c}
u h n_{x}+v h n_{y} \\
\left(u^{2} h+g\left[\zeta^{2}+2 \zeta h_{s}\right] / 2\right) n_{x}+u v h n_{y} \\
u v h n_{x}+\left(v^{2} h+g\left[\zeta^{2}+2 \zeta h_{s}\right] / 2\right) n_{y}
\end{array}\right]
$$

and

$$
\mathbf{f}^{v}=\left[\begin{array}{c}
0 \\
(h \partial u / \partial x) n_{x}+(h \partial u / \partial y) n_{y} \\
(h \partial v / \partial x) n_{x}+(h \partial v / \partial y) n_{y}
\end{array}\right]
$$


where $n_{x}$ and $n_{y}$ are the Cartesian components of $\mathbf{n}$, the unit normal vector to $S$.

\section{Inviscid fluxes}

The inviscid fluxes can be expressed by adopting Roe's approximate Riemann solver at the cell interface as follows

$$
\mathbf{f}^{\mathbf{1}}{ }_{i, j}=\frac{1}{2}\left[\mathbf{f}^{\mathbf{1}}\left(\mathbf{q}_{i, j}^{+}\right)+\mathbf{f}^{\mathbf{I}}\left(\mathbf{q}_{i, j}^{-}\right)-|\mathbf{A}|\left(\mathbf{q}_{i, j}^{+}-\mathbf{q}_{i, j}^{-}\right)\right]
$$

with

$$
|\mathbf{A}|=\mathbf{R}|\Lambda| \mathbf{L}
$$

where $\mathbf{q}_{i, j}^{+}$and $\mathbf{q}_{i, j}^{-}$are reconstructed right and left Riemann states at the cell interface, respectively, located between adjacent cells $i$ and $j, \mathbf{R}$ and $\mathbf{L}$ the right and left eigenvector matrices of flux Jacobian A respectively, and $|\Lambda|$ is a diagonal matrix of the absolute values of the eigenvalues of $\mathbf{A}$. The inviscid flux Jacobian is given by

$$
\begin{aligned}
\mathbf{A} & =\frac{\partial\left(\mathbf{f}^{\mathbf{I}}\right)}{\partial \mathbf{q}} \\
& =\left[\begin{array}{ccc}
0 & n_{x} & n_{y} \\
\left(c^{2}-u^{2}\right) n_{x}-u v n_{y} & 2 u n_{x}+v n_{y} & u n_{y} \\
-u v n_{x}+\left(c^{2}-u^{2}\right) n_{y} & v n_{x} & u n_{x}+2 v n_{y}
\end{array}\right]
\end{aligned}
$$

which has eigenvalues given by

$$
\begin{aligned}
& \lambda_{1}=u n_{x}+v n_{y} \\
& \lambda_{2}=u n_{x}+v n_{y}-c \\
& \lambda_{3}=u n_{x}+v n_{y}+c
\end{aligned}
$$

The associated right eigenvector matrix is

$$
\mathbf{R}=\left[\begin{array}{ccc}
0 & 1 & 1 \\
n_{y} & u-c n_{x} & u+c n_{x} \\
-n_{x} & v-c n_{y} & v+c n_{y}
\end{array}\right]
$$

and the left eigenvector matrix is given by

$$
\mathbf{L}=\left[\begin{array}{ccc}
-\left(u n_{y}-v n_{x}\right) & n_{y} & n_{x} \\
\frac{1}{2}+\frac{u n_{x}+v n_{y}}{2 c} & \frac{-n_{x}}{2 c} & \frac{-n_{y}}{2 c} \\
\frac{1}{2}-\frac{u n_{x}+v n_{y}}{2 c} & \frac{n_{x}}{2 c} & \frac{n_{y}}{2 c}
\end{array}\right]
$$

The variables $u, v$, and $c$ in (9) through (12) are evaluated by Roe's average state which is defined as

$$
u=\frac{u^{+} \sqrt{h^{+}}+u^{-} \sqrt{h^{-}}}{\sqrt{h^{+}}+\sqrt{h^{-}}}, v=\frac{v^{+} \sqrt{h^{+}}+v^{-} \sqrt{h^{-}}}{\sqrt{h^{+}}+\sqrt{h^{-}}}
$$

and

$$
c=\sqrt{\frac{g\left(h^{+}+h^{-}\right)}{2}}
$$

where the superscripts + and - denote the left and right Riemann states on either side of a cell interface, respectively.

\section{Slope limiter}

Spatial information on the variables within the cell can be obtained from an interpolation across an arbitrary cell $A$ given by

$$
\mathbf{q}(x, y)=\mathbf{q}_{A}+\left(\nabla \cdot \mathbf{q}_{A}\right) \mathbf{r}
$$

where $\mathbf{q}_{A}$ is the value of $\mathbf{q}$ at the centre of cell $A, \nabla$ the gradient vector, and $\mathbf{r}(x, y)$ the vector from the cell centre to any arbitrary point within the cell. (14) is a piecewise linear interpolation which gives a second order spatial approximation.

However, numerical oscillations can occur when evaluating $\nabla \cdot \mathbf{q}_{A}$ without using limiters. The limiters suppress the generation of local extrema in a non-linear manner when the variables are reconstructed. Herein, the minmod limiter (Toro, 1999) is employed for all test cases. It is expressed for consecutive cells $(i-1),(i)$, and $(i+1)$ in $x$-direction as follows,

$$
\begin{aligned}
& \mathrm{q}(x)=\mathrm{q}_{i}+\frac{\Phi}{2}\left(\mathrm{q}_{i}-\mathrm{q}_{i-1}\right) \\
& \Phi(r)=\max [0, \min (r, 1)]
\end{aligned}
$$

and

$$
r=\frac{q_{i+1}-q_{i}}{q_{i}-q_{i-1}}
$$

\section{Open boundary conditions}

Riemann invariants, specified according to the local Froude number, have been used to implement open boundary conditions as follows:

(i) subcritical flow (when the water depth $h_{B}$ is imposed)

$$
U_{B}=U_{I}+2 \sqrt{g}\left(\sqrt{h_{I}}-\sqrt{h_{B}}\right), \quad V_{B}=V_{I}
$$

(ii) subcritical flow (when the velocity $U_{B}$ is imposed)

$$
h_{B}=\left(\frac{1}{2 \sqrt{g}}\left(U_{l}-U_{B}\right)+\sqrt{h_{l}}\right)^{2}, \quad V_{B}=V_{l}
$$

(iii) supercritical inflow (the variables $\left(h_{B}, U_{B}, V_{B}\right)$ are given)

and 
(iv) supercritical outflow

$$
h_{B}=h_{l}, \quad U_{B}=U_{l}, \quad V_{B}=V_{I}
$$

Subscript $B$ denotes the boundary value, subscript $I$ denotes the inner Riemann state value at the boundary, and $U$ and $V$ the depth-averaged velocity components normal and tangential to the boundary, respectively.

\section{Hydraulic jump}

Using the data of hydraulic jump experiments conducted by Gharangik and Chaudhry (1991), the accuracy of the model presented is demonstrated. The hydraulic jump was formed in a horizontal $14 \mathrm{~m}$ long and $0.46 \mathrm{~m}$ wide rectangular metal flume. Two cases, whose data of the results were documented in their paper, were used here for validation tests. The flow conditions of the tests are shown in Table 1.

Since one-dimensional approach is enough to simulate the hydraulic jump in this case, we here consider $x$ direction only. After Gharangik and Chaudhry (1991), the bottom friction term in (2) is expressed by employing Manning coefficient as

Table 1: Flow conditions of hydraulic jump experiments

\begin{tabular}{ccccc}
\hline $\begin{array}{c}\text { Test } \\
\text { name }\end{array}$ & $\begin{array}{c}\text { Upstream } \\
\text { depth }(\mathrm{m})\end{array}$ & $\begin{array}{c}\text { Velocity } \\
(\mathrm{m} / \mathrm{s})\end{array}$ & $\begin{array}{c}\text { Froude } \\
\text { number }\end{array}$ & $\begin{array}{c}\text { Downstream } \\
\text { depth }(\mathrm{m})\end{array}$ \\
\hline $\mathrm{A}$ & 0.024 & 3.255 & 6.65 & 0.195 \\
$\mathrm{~B}$ & 0.043 & 2.737 & 4.23 & 0.222 \\
\hline
\end{tabular}

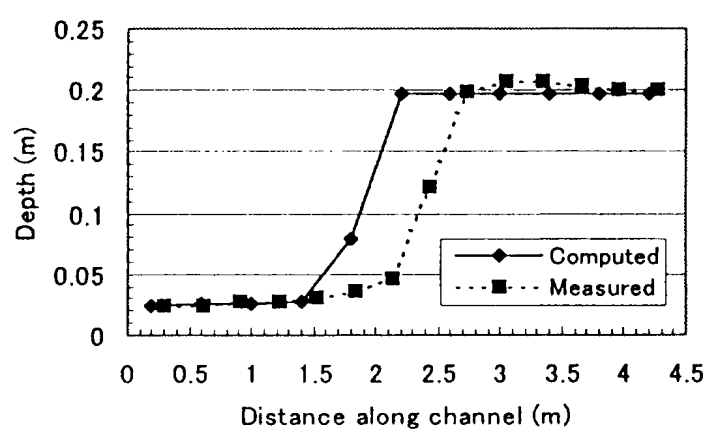

Figure 1: Jump profile for case $A(F r=6.65)$

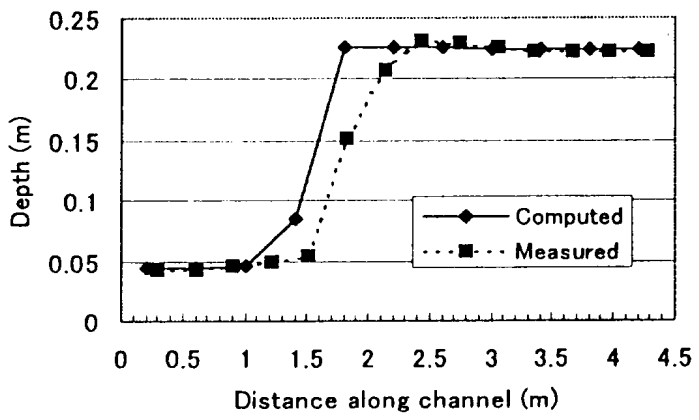

Figure 2: Jump profile for case $\mathrm{B}(\mathrm{Fr}=4.23)$

$$
\frac{\tau_{b x}}{\rho}=\frac{n^{2} g u|u|}{h^{1 / 3}}
$$

where $n$ is Manning coefficient.

Time increment was set to $0.05 \mathrm{~s}$ and the length of computational grid was $0.4 \mathrm{~m}$. Manning coefficient was determined by trial and error by matching the computed location of the hydraulic jump with the measured location of that. The resulting Manning coefficients in case $A$ and $B$ were 0.0152 and 0.0145 , respectively. The viscous terms were ignored in both cases. It took about $250 \mathrm{~s}$ to reach steady state in these runs. The results are shown in Figure 1 and Figure 2 for the case of A and B, respectively. Comparing the computed and measured water surface profiles, the computed results are in good agreement with the measured results. Especially, the water depths in front of and behind the hydraulic jump are well reproduced. However, the computed locations of hydraulic jump are slightly different from the measured ones.

\section{Tidal wave propagation}

This test case is for checking the model presented to work properly in a channel with a variable depth (Bermudes and Vazquez, 1994). Since the channel can be treated as one dimensional, we also consider about $x$ direction only in this case. The initial water depth and boundary conditions are given as follows, and at the same time they are depicted in Figure 3.

$$
\begin{gathered}
H(x)=50.5-\frac{40 x}{L}+10 \sin \left\{\pi\left(\frac{4 x}{L}+\frac{1}{2}\right)\right\} \\
\zeta(0, t)=4-4 \sin \left\{\pi\left(\frac{4 t}{86400}-\frac{1}{2}\right)\right\} \\
u(x, 0)=0
\end{gathered}
$$

and

$$
u(L, t)=0
$$

where $L(=648,000 \mathrm{~m})$ is the length of the channel and $x$ is the distance from the mouth of the channel. (18) expresses a tidal wave of $4 \mathrm{~m}$ amplitude. In this case, the time step was set to $30 \mathrm{~s}$ and the length of numerical grid was $2,700 \mathrm{~m}$. To eliminate any damping effect, both the coefficients of kinematic eddy and bottom friction were set to zero. The simulation was undertaken for $864,000 \mathrm{~s}$. The computed free surface elevation and discharge are shown in Figure 4 and Figure 5, respectively. The computed free surface elevation declines from $4 \mathrm{~m}$ to $0 \mathrm{~m}$ almost linearly, as the distance increases from $0 \mathrm{~m}$ to about $200 \mathrm{~km}$. Similarly, the discharge decreases from about $93 \mathrm{~m}^{3} / \mathrm{s}$ to $0 \mathrm{~m}^{3} / \mathrm{s}$ linearly. In the inner region from $200 \mathrm{~km}$ to the other end of the channel, the water still has no motion. These results agree very well with those of Bermudez and Vazquez (1994). This shows that (2) is discretized properly in the model. 


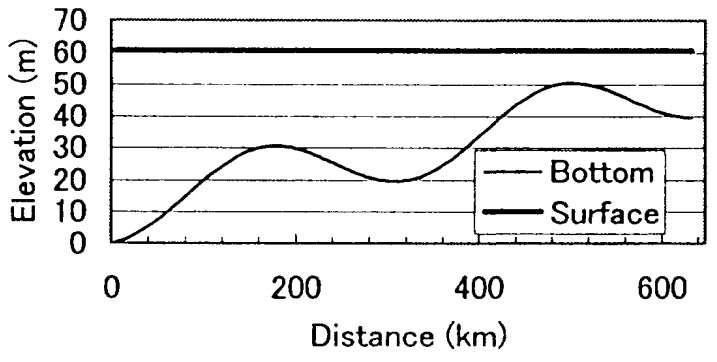

Figure 3: Bottom configuration and initial water surface

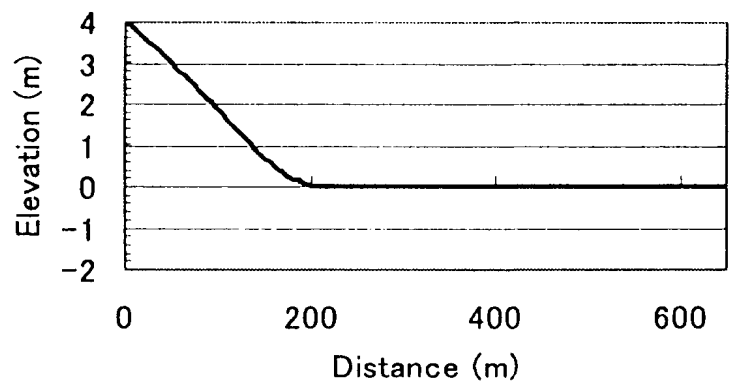

Figure 4: Water surface elevation at $t=864,000 \mathrm{~s}$

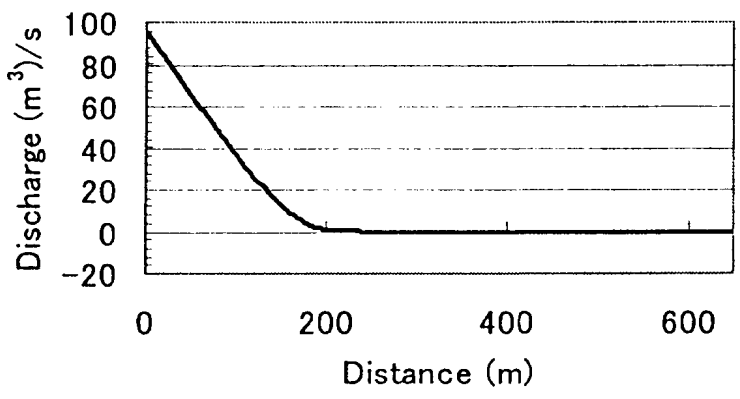

Figure 5: Discharge at $t=864,000 \mathrm{~s}$

10 Flow over a cascade-type head work

A cascade type head work was introduced when the old head work constructed across the Okitsu river in Shizuoka prefecture was repaired (Suto, 1998). This improvement aimed to create a fish-friendly weir, as well as an amenity space in the river. Three stages of artificial cascade across the river were constructed. Taking into consideration of jumping ability of ayu, Plecoglossus alitivelis, the height of a cascade was decided to 80 $\mathrm{cm}$ and each stage has a pool with about $50 \mathrm{~cm}$ depth. This also would provide children with space, where they can safely play with water.

A model experiment of this cascade type head work was conducted by Kojima and Yamamoto (1999). In their experiment, a 1:6 scale model of the prototype was installed in a flume. The scale of one stage of the model is depicted in Figure 6. Since the head work crosses the river with uniform configuration, one dimensional approach is also valid in this case. The length of computational grid was set to $0.0234 \mathrm{~m}$ and time step was $0.001 \mathrm{~s}$. The coefficient of kinematic eddy viscosity was $0.1 \mathrm{~m}^{2} / \mathrm{s}$ and the bottom friction was ignored. The water depth at the upstream was set to $0.155 \mathrm{~m}$ as a boundary condition. The simulation was undertaken when the discharge was $30 \mathrm{~L} / \mathrm{s}$, which corresponds to the design discharge of this weir. It took about $15 \mathrm{~s}$ to reach steady state.

The computed water surface, velocity, and Froude number are shown in Figures 7,8 , and 9, respectively. Figure 9 shows that the flow is subcritical in pools and supercritical on the slope of cascades. Over $1.5 \mathrm{~m} / \mathrm{s}$ velocity was computed at the second cascade and the velocity on the fourth slope of cascade was smallest compared to others on the slope. As water flows down, the velocity on the slope of cascade decreases.

Since the detailed data of experiments were not documented in the paper, the measured data cannot be depicted in Figure 7. However, judging from the figures depicted in the paper, the computed gradient of water surface, where a hydraulic jump is supposed to occur, seems to be higher than the measured data. This is mainly due to the flow condition in the pools. In the experiment, the directions of the flow in the upper and lower layers near the head of pool were supposed to be opposite, and horizontal eddies were formed. However, this flow condition cannot be reproduced by using shallow water equations, which assume the same flow direction from the bottom to the surface. In order to overcome this discrepancy, vertically two-dimensional equations should be used as governing equations, and at the same time a proper turbulent model should be employed to evaluate the Reynolds stress in and around a hydraulic jump.

\section{Flow through a vertical slot type fishway}

As the concern for conserving or improvement of fishfriendly rivers has been widespread, fishways have attracted major interests. Simulating the flow in fishways is crucially important, when designing them. Ideally, the configuration of a fishway is adjusted to the form suitable for the target fish, considering the computed flow properties.

A vertical slot type fishway is one of the popular fishways. It can be installed where the river discharge largely changes. The configuration used in this test is depicted in Figure 10. Figure 11 shows a corresponding non-uniform quadtree grid used for the present simulation where the smallest cells are of subdivision level 9 corresponding to a space square grid $0.0195 \mathrm{~m}$. The bottom gradient was set to $1 / 15$. The coefficient of kinematic eddy viscosity was modelled by Smagorinsky (1963). It is expressed in two-dimensional form as follows.

$$
v=(c \Delta)^{2}\left\{2\left(\frac{\partial u}{\partial x}\right)^{2}+2\left(\frac{\partial v}{\partial y}\right)^{2}+\left(\frac{\partial v}{\partial x}+\frac{\partial u}{\partial y}\right)^{2}\right\}^{\frac{1}{2}}
$$

where $\Delta$ is the length of the grid side and $c(=0.1)$ is constant. The bottom friction was ignored. Both the upper and lower stream depths were set to be constant 


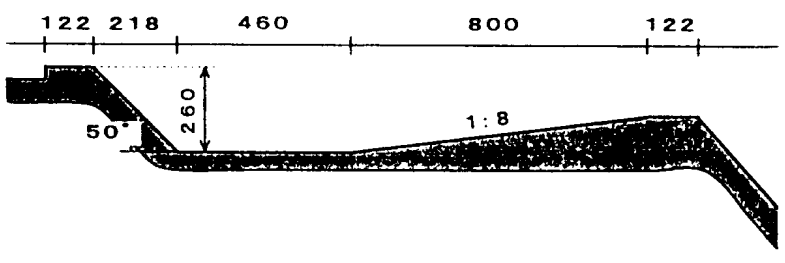

Figure 6: Diagram of one stage of cascade model (unit: $\mathrm{mm}$ )

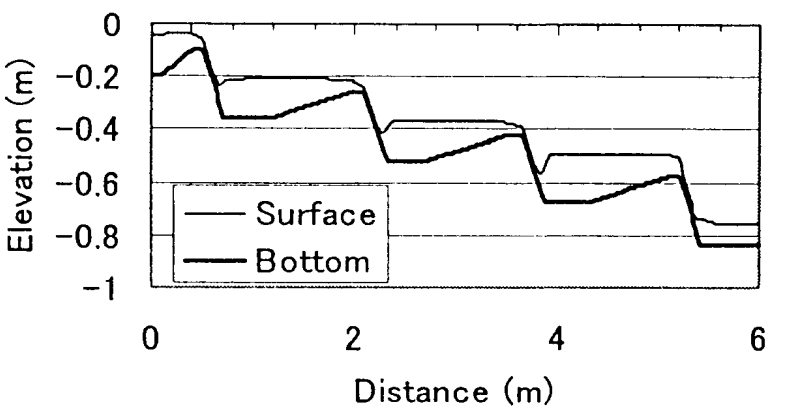

Figure 7: Bottom topography and water surface

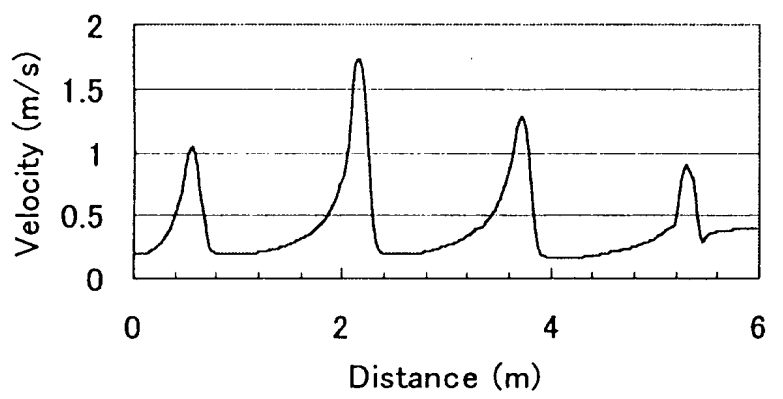

Figure 8: Velocity distribution

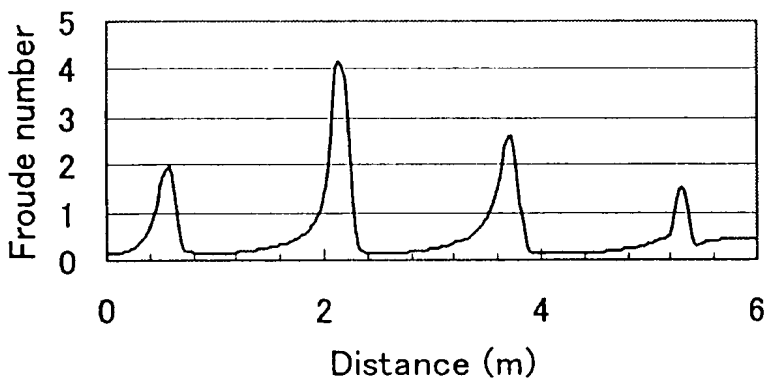

Figure 9: Froude number

as $0.5 \mathrm{~m}$, and time step was taken as $0.001 \mathrm{~s}$. The resultant discharge was $0.191 \mathrm{~m}^{3} / \mathrm{s}$.

The computed results are visualised in Figures 12 through 14. The velocity vector field is shown in Figure 12. The maximum velocity is $2.4 \mathrm{~m} / \mathrm{s}$. The water flowing through a slot gradually turns left as the water flows and heads for the next slot, making an anticlockwise eddy at the left side of the main stream flow. The water flows upstream along the right-hand wall of a pool. These flow properties agree well with the measured ones (Rajaratnam et al., 1998).

Figure 13 shows equi-velocity lines. The main stream from one slot to the next can easily be seen in Figure 13. Since the velocity in the slot is about $1.5 \mathrm{~m} / \mathrm{s}$ in this discharge, the target fish should have enough ability to swim with the over $1.5 \mathrm{~m} / \mathrm{s}$ speed. In the main stream, the water flows with the speed of over $1 \mathrm{~m} / \mathrm{s}$.

The equi-Froude number lines with an interval of 0.1 are shown in Figure 14. Supercritical flow is occurring in slots with the maximum Froude number of 2.93. In most part of the fishway, the Froude number is less than 0.5 and thus the flow is in subcritical.

\section{Conclusions}

A second-order accurate Godunov-type solver of the shallow water equations in finite volume form on quadtree grids is described. The model presented accounts properly for the spatially-varying bathymetry and is suitable for Roe's approximate Riemann scheme.

Results are presented for several validation tests, which highlight certain features of the present shallowflow solver. To make sure that the model could capture a discontinuous front clearly, the numerical test for a hydraulic jump was conducted. Then, the effect of nonuniform bed conditions was examined by simulating tidal wave propagation into a channel. In order to demonstrate that the model could be a useful tool in designing the river structure, the flows over a cascade-type head work and through a vertical slot type fishway were simulated.

Taking into consideration the accuracy of computed results presented in this work, the model presented will be a very useful tool for intimate river works for nature conservation, which are becoming very popular in these days.

\section{Acknowledgements}

The author wishes to express his sincere gratitude to Dr. A. G. L. Borthwick and Mr. B. Rogers of University of Oxford for helping computer program coding. Thanks are also due to Professor T. Kawachi of Kyoto University for encouraging completing this paper.

\section{References}

[1] Alcrudo, F. and Garcia-Navarro, P. (1993): A highresolution Godunov-type scheme in finite volumes for the 2D shallow-water equations, Int. J. Numer. Methods Fluids, 16, pp.489-505.

[2] Anastasiou, K. and Chan, C. T. (1997): Solution of the $2 \mathrm{D}$ shallow water equations using the finite volume method on unstructured triangular meshes, Int. J. $\mathrm{Nu}$ mer. Methods Fluids, 24, pp.1225-1245.

[3] Bermudes, A. and Vazquez, M. E. (1994): Upwind methods for hyperbolic conservation laws with source terms, Computers Fluids, 23(8), pp. 1049-1071.

[4] Borthwick, A. G. L. and Akponasa G. A. (1997): Reservoir flow prediction by contravariant shallow water equations, J. Hydr Engrg, ASCE, 123(5), pp.432-439.

[5] Gharangik, A. M. and Chaudhry, M. H. (1991): Numerical simulation of hydraulic jump, J. Hydr. Engrg., ASCE, 117(9), pp.1195-1211. 

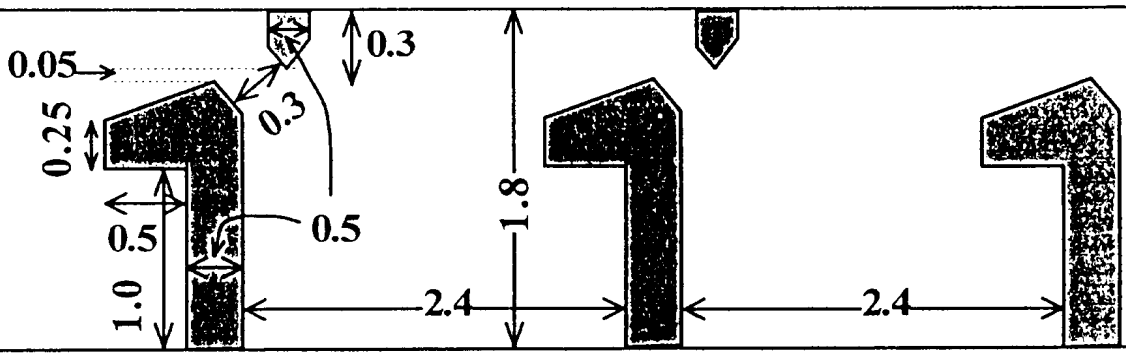

(․․

Figure 10: Diagram of a vertical slot type fishway (unit: $\mathrm{m}$ ) The bottom gradient is $1 / 15$.

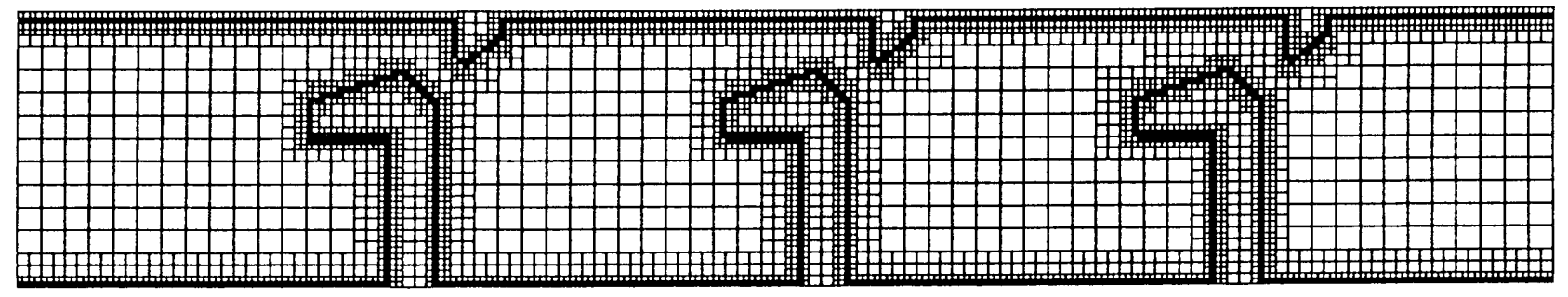

Figure 11: Quadtree grids for a vertical slot type fishway

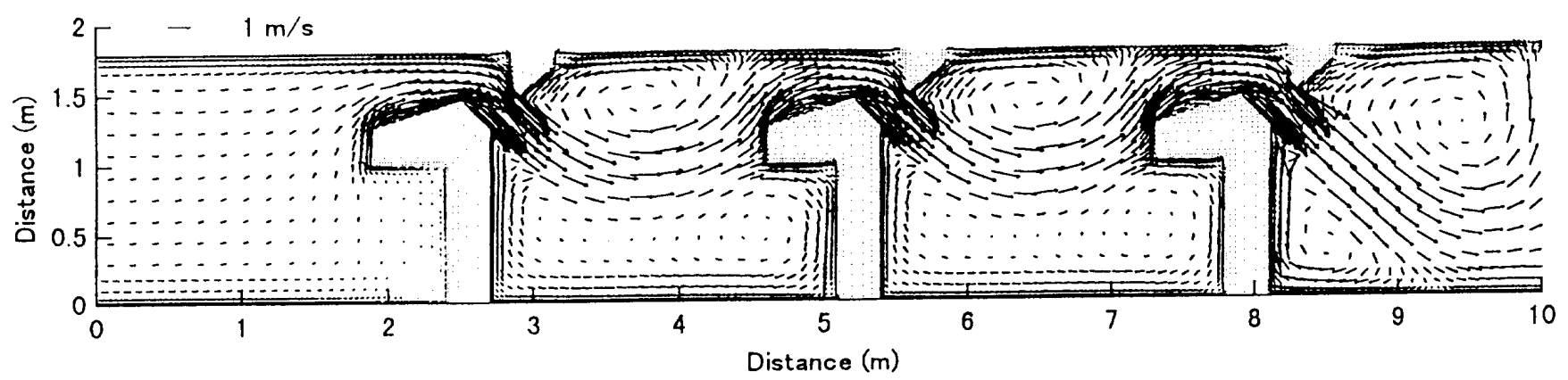

Figure 12: Velocity vector field

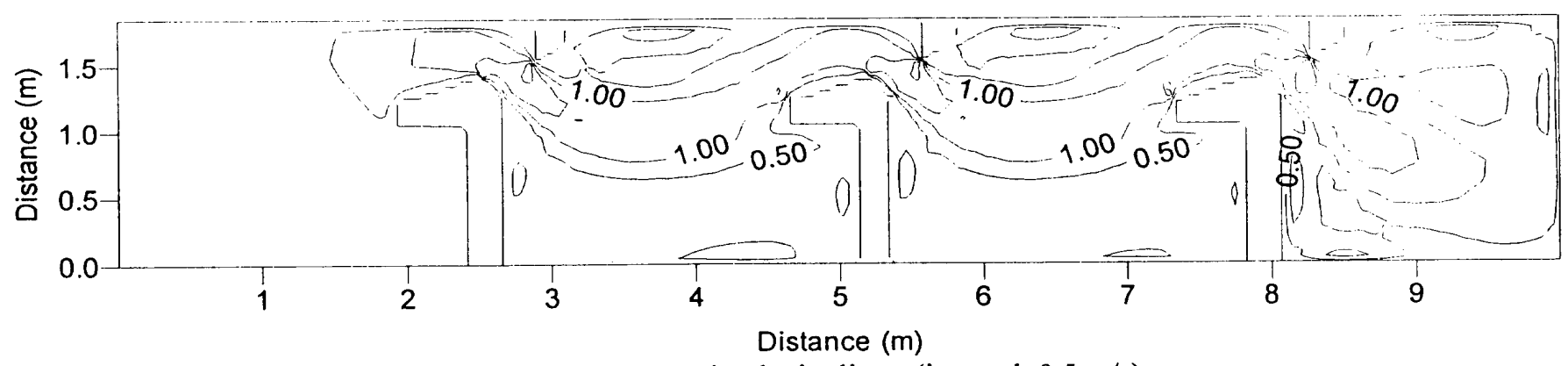

Figure 13: Equi-velocity lines (interval: $0.5 \mathrm{~m} / \mathrm{s}$ )

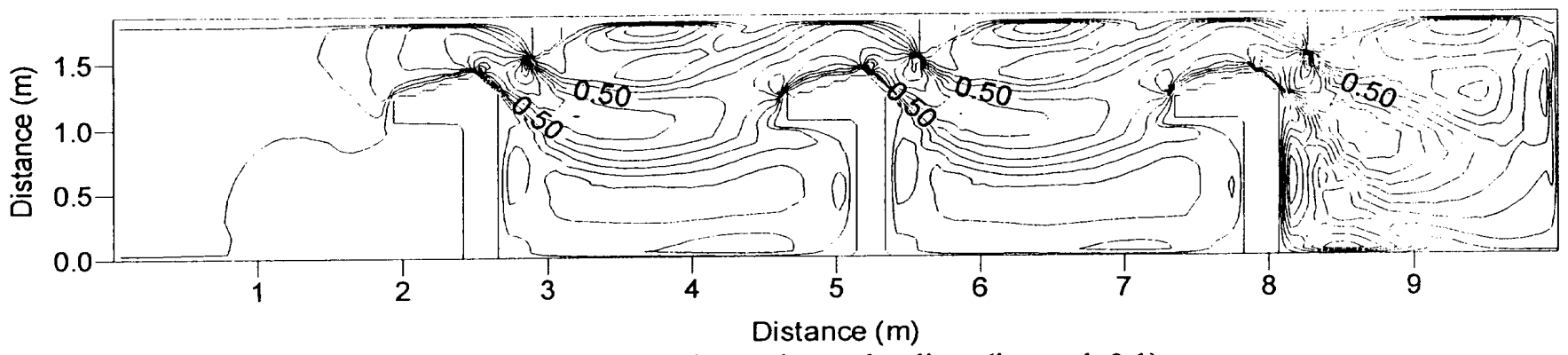

Figure 14: Equi-Froude number lines (interval: 0.1) 
[6] Greaves, D. M. and Borthwick, A. G. L. (1998): On the use of adaptive hierarchical meshes for numerical simulation of separated flows, Int. J. Numer. Methods Fluids, 26, pp.303-322.

[7] Hu, K., Mingham, C. G., and Causon, D. M. (1998): A bore-capturing finite volume method for open-channel flows, Int. J. Numer. Methods Fluids, 28, pp.12411261.

[8] Kojima, N. and Yamamoto, M.(1999): Flow in water cushion of cascade type head work, Proc. Annual Meeting of Japanese Soc. Irrig. Drng. Recl. Engrg, pp.50-51. (in Japanese).

[9] Lin, B. and Falconer, R. A. (1995): Modelling sediment fluxes in estuarine waters using curvilinear coordinate system, Estuary, Coastal and Shelf Science, 14, pp.413-428.

[10] Mingham, C. G. and Causon, D. M. (1998): Highresolution finite-volume method for shallow water flows, J. Hydr. Engrg., ASCE, 124(6), pp.605-614.

[11] Nujic, M. (1995): Efficient implementation of nonoscillatory schemes for the computation of freesurface flows, J. Hydr. Res., 33(1), pp.101-111.

[12] Rajaratnam, N., Vinne, G. V., and Katopodis, C. (1986): Hydraulics of vertical slot fishways, J. Hydr. Engrg., ASCE, 112(10), pp.909-927.
[13] Roe, P. L. (1981): Approximate Riemann Solvers, Parameter Vectors, and Difference Schemes, J. Comp. Phys., 43, pp.357-372.

[14] Rogers, B., Fujiahra, M., and A. G. L. Borthwick: Adaptive Q-tree Godunov-type scheme for Shallow Water Equations', Int. J. Numer. Methods Fluids, (in press).

[15] Suto T.(1998): Cascade type head work - case of Wadashima -, J. Japanese Soc. Irrig. Drng. Rrecl. Engrn, 66(11), pp.61-66. (in Japanese).

[16] Smagorinsky, J. (1963): General circulation experiments with the primitive equations, Mon. Weath. Rev.,93 , pp.99-165.

[17] Toro, E. F. (1999): Riemann solvers and numerical methods for fluid dynamics - A practical introduction, Springer, pp.341-488.

[18] Zhao, D. H., Shen, H. W., Lai, J. S., and Tabios III, G. Q.(1996): Approximate Riemann solvers in FVM for 2D hydraulic shock wave modeling, J. Hydr. Engrg., $A S C E$, 122(12), pp.692-702.

[19] Zhao, D. H., Shen, H. W., Tabios III, G. Q., Lai, J. S., and Tan, W. Y. (1994): Finite-volume two-dimensional unsteady-flow model for river basins, J. Hydr. Engrg., ASCE, 120(7), pp.863-883. 